\title{
CONTACT STRUCTURES, FOLIATIONS, AND THE FUNDAMENTAL GROUP
}

\author{
N. A'CAMPO AND D. KOTSCHICK
}

\section{Introduction}

It was proved in [12] that every finitely presentable group is the fundamental group of an orientable closed 5-manifold which admits an almost contact structure. In Section 2 of this paper we use a recent result of A. Weinstein [18] to prove the following stronger result.

THEOREM 1. Every finitely presentable group is the fundamental group of an orientable closed 5-dimensional contact manifold.

The corresponding result is trivial in dimensions $2 n-1 \geqslant 7$, because for $n \geqslant 4$ every finitely presentable group can be realized as the fundamental group of an orientable closed $n$-manifold $M$. The unit cotangent bundle of an arbitrary Riemannian metric on $M$ has the same fundamental group and a tautological contact structure.

Thinking of a contact structure as a completely non-integrable hyperplane distribution, there is a question dual to the one answered above: Does the existence of an integrable $k$-dimensional distribution, that is, a foliation, on an orientable closed $n$-manifold $M$ restrict the fundamental group of $M$, or not? The answer is as follows.

THEOREM 2. If $n \geqslant 5$ and $n \geqslant k \geqslant 0$ or $n=4$ and $k \in\{0,2,4\}$, then every finitely presentable group is the fundamental group of an orientable closed n-dimensional manifold with a k-dimensional foliation of class $C^{\infty}$ with trivial Godbillon-Vey invariant.

The proof of Theorem 2, given in Section 3, is elementary and explicit in all cases. Even in the codimension 1 case we do not use Thurston's deep existence theorem [17]. As with Theorem 1, the result is trivial in dimensions $n \geqslant 7$, and the interest lies in sorting out the low-dimensional cases.

Theorem 2 is sharp in several respects, as follows.

REMARK 1.1. If one requires the foliations to be analytic rather than smooth, then a result of A. Haefliger [8] shows that the fundamental group is restricted for $k=n-1$, in that no finite groups are possible.

\section{Received 20 July 1992.}

1991 Mathematics Subject Classification 57M05.

This paper was presented at the Joint Meeting of the American and London Mathematical Societies in Cambridge, June/July 1992. 
REMARK 1.2. Considering smooth foliations defined globally by a closed differential form restricts the fundamental group if $k=n-1$, because in that case the first Betti number is positive. (We owe this remark to A. Haefliger.)

REMARK 1.3. A closed oriented $n$-manifold carries a $k$-dimensional foliation for $k=1$ or $n-1$ only if its Euler characteristic vanishes. For $n=4$ this imposes nontrivial restrictions on the fundamental group. Again, the first Betti number has to be positive, but this is not sufficient; compare [10].

The results of this paper (and of $[16,18]$ ) can be thought of as an instance of Gromov's Homotopy Principle [7] applied to contact structures and to foliations.

For orientable closed manifolds of dimension 3, not all finitely presentable groups arise as fundamental groups. However, those which do arise do not obstruct the existence of contact structures [14] or of foliations [13]. Our proof of Theorem 1 is a generalization of J. Martinet's argument [14] for the 3-dimensional case.

\section{Proof of Theorem 1}

Recall that a submanifold $Y$ of a contact manifold $X$ is said to be isotropic if its tangent bundle is contained in the contact distribution. The contact distribution has a natural conformal symplectic structure, and we denote by $\perp$ the symplectic orthogonal with respect to this structure. If $Y$ is isotropic, then $T Y \subset(T Y)^{\perp}$, so that the conformal symplectic normal bundle $\operatorname{CSN}(X, Y)=(T Y)^{\perp} / T Y$ is defined. Weinstein [18] proved that the manifold obtained from $X$ by surgery on an isotropic $k$-sphere with trivialized conformal symplectic normal bundle is again a contact manifold.

Now let $\Gamma$ be a finitely presented group. For each generator we take a copy of $S^{1} \times S^{4}$ with its standard contact structure, and form the connected sum $X$ as a contact manifold using the case $k=0$ of Weinstein's theorem, or [15]. Each relation of the presentation corresponds to a unique homotopy class of a map $S^{1} \rightarrow X$, and we can represent these classes by disjoint embedded circles. Let $Y$ be the union of these circles. According to the lemma below, we may assume that $Y$ is an isotropic submanifold. The conformal symplectic normal bundle $\operatorname{CSN}(X, Y)$ is of rank 2, and it follows from the connectedness of $\mathrm{Sp}(2)$ that it is trivial. Performing surgery and using the case $k=1$ of Weinstein's theorem, we obtain an orientable contact 5manifold with fundamental group $\Gamma$.

This completes the proof of Theorem 1 modulo the following lemma.

LEMMA 2.1. Let $X$ be an orientable contact manifold, and $\phi: S^{1} \rightarrow X$ an embedding. Then $\phi$ is isotopic to an embedding with isotropic image.

Proof. The case $\operatorname{dim} X=3$ is a special case of Lemma 2.2.1 in [2].

The general case can be reduced to this as follows. Let $\alpha$ be a contact form on $X$. Choose a vector field $v$ along $\phi$ such that $\alpha_{\phi(t)}\left(v_{\phi(t)}\right)=1$, with $v_{\phi(t)}$ and $\dot{\phi}(t)$ linearly independent for all $t \in S^{1}$. Choose a second vector field $w$ along $\phi$ such that $\alpha_{\phi(t)}\left(w_{\phi(t)}\right)=0$ and

$$
(d \alpha)_{\phi(t)}\left(w_{\phi(t)}, \dot{\phi}(t)-(\alpha(\dot{\phi}(t))) v_{\phi(t)}\right)=1
$$


Now let $M$ be a 3-dimensional submanifold of a tubular neighbourhood of $\phi\left(S^{1}\right)$ in $X$, with $\phi\left(S^{1}\right) \subset M$ and $T_{\phi(t)} M=\operatorname{Span}\left\{v_{\phi(t)}, w_{\phi(t)}, \dot{\phi}(t)\right\}$. After shrinking $M$, if necessary, $\left.\alpha\right|_{M}$ is a contact form on $M$. An isotopy of $\phi$ in $M$ to an embedding with isotropic image exists by [2] and produces the desired isotopy.

REMARK 2.1. Instead of using Weinstein's theorem [18], we could phrase the argument in the framework of Eliashberg [3]. This is completely equivalent, and shows that the contact structures so constructed are holomorphically fillable.

REMARK 2.2. After we had found the above proof of Theorem 1, R. E. Gompf [6], in reply to a question from the authors, proved the analogous theorem for symplectic 4-manifolds. That case is much more difficult. Using the argument of [12], it yields another proof of Theorem 1 .

\section{Proof of Theorem 2}

All the manifolds considered in this section are orientable, closed and smooth.

Let $\Gamma$ be a finitely presentable group. We shall say that $\Gamma$ is $(n, k)$-realizable if it is (isomorphic to) the fundamental group of an $n$-manifold with a smooth $k$-dimensional foliation. Each of the cases in Theorem 2, except the trivial ones $(k \in\{0, n\})$, is covered by at least one of the following five existence lemmas. Lemma 3.6 proves that the foliations constructed have trivial generalized Godbillon-Vey invariants, completing the proof of Theorem 2 .

LEMMA 3.1. If $n \geqslant 5$, then $\Gamma$ is $(n, 1)$-realizable.

Proof. One can easily construct an $n$-manifold $X$ with fundamental group $\pi_{1}(X) \cong \Gamma$ and Euler characteristic $\chi(X)=0$. Then, by the Poincaré-Hopf theorem, $X$ has a vector field without zeros. Its integral curves yield the desired foliation.

LEMMA 3.2. If $n \geqslant 7$ and $2 \leqslant k \leqslant n-2$ or $n=6$ and $k \in\{2,4\}$, then $\Gamma$ is $(n, k)$ realizable by a product foliation.

Proof. The assumptions imply $m=\max \{k, n-k\} \geqslant 4$ and $\min \{k, n-k\} \geqslant 2$. Thus we can find an $m$-manifold $X$ with $\pi_{1}(X) \cong \Gamma$, such that $X \times S^{n-m}$ shows that $\Gamma$ is $(n, k)$-realizable by a product foliation.

LEMMA 3.3. If $n \geqslant 5$, then $\Gamma$ is $(n, n-3)$-realizable.

Proof. Let $X$ be a 4-manifold with $\pi_{1}(X) \cong \Gamma$. By replacing $X$ with its connected sum with a number of copies of $\mathbb{C} P^{2}$ if necessary, we may assume $\chi(X)>0$ and $\operatorname{rank} \pi_{2}(X) \geqslant n-4$.

The manifold $X$ has a vector field $v$ with zeros $P_{1}, P_{2}, \ldots, P_{\chi}$, all of index +1 . Let $D_{1}^{4}, D_{2}^{4}, \ldots, D_{\chi}^{4}$ be a collection of small disjoint disks centred at the points $P_{i}$. We identify these with the standard unit disk in $\mathbb{C}^{2}$ and denote by $S_{i}^{3} \subset D_{i}^{4}$ the sphere of radius $\frac{1}{2}$. In the outside collar of $S_{i}^{3}$ we can deform the vector field $v$ to make its flow lines asymptotic to the fibres of the Hopf fibration of $S_{i}^{3}$. Doing this near each $S_{i}^{3}$ produces a non-singular 1-dimensional foliation $\mathscr{F}$ on the complement of the disks of radius $\frac{1}{2}$ around the zeros of $v$, tangent and restricting to the Hopf fibration on each boundary component. 
The complex coordinates around $P_{i}$ can be used to 'blow up' these points in a standard way. This replaces a disk of radius $\frac{1}{2}$ around $P_{i}$ by a $D^{2}$-bundle $E_{i}$ over $S^{2}$. This does not change the fundamental group, because topologically it amounts to forming the connected sum with one copy of $\overline{\mathbb{C} P^{2}}$ at each $P_{i}$. We now replace $X$ with this connected sum.

Let $\pi: Y \rightarrow X$ be an $S^{1}$-bundle which has degree 1 on one 2-sphere free in $\pi_{2}(X)$ and is trivial otherwise. The exact sequence of homotopy groups induced by $\pi$ shows $\pi_{1}(Y) \cong \pi_{1}(X)(\cong \Gamma)$. The pullback $\pi^{*}(\mathscr{F})$ is a 2 -dimensional foliation on $Y \backslash\left(\bigcup\left(E_{i} \times S^{1}\right)\right)$, tangent and restricting to the product of the Hopf fibration with $S^{1}$ on each boundary component. One can fill in standard Reeb foliations on each fibre $D^{2} \times S^{1}$ of each $E_{i} \times S^{1}$ to obtain a smooth 2-dimensional foliation on $Y$. This shows that $\Gamma$ is $(5,2)$-realizable.

We have $\operatorname{rank} \pi_{2}(Y)=\operatorname{rank} \pi_{2}(X)-1 \geqslant n-5$. If $n>5$, we can take another circle bundle $Z \rightarrow Y$ with $\pi_{1}(Z)=\pi_{1}(Y)$ and $\operatorname{rank} \pi_{2}(Z)=\operatorname{rank} \pi_{2}(Y)-1$, and pull back the foliation. Iteration shows that $\Gamma$ is $(n, n-3)$-realizable.

\section{LEMMA 3.4. If $n \geqslant 4$, then $\Gamma$ is $(n, n-2)$-realizable.}

Proof. Let $X$ be an oriented 4-manifold with $\pi_{1}(X) \cong \Gamma$, and $\bar{X}$ the same manifold with the opposite orientation. It was proved in [11] that there exists an $a$ such that the connected sum $Y=X \# a \mathbb{C} P^{2}$ has an almost complex structure. Applying the same argument to $\bar{Y}$, we find that there is a $b$ such that $Z=X \# a \mathbb{C} P^{2} \# b \overline{\mathbb{C} P^{2}}$ and $\bar{Z}$ both have almost complex structures. (The construction of the second structure does not destroy the first one because forming the connected sum with $\overline{\mathbb{C} P^{2}}$, unlike with $\mathbb{C} P^{2}$, is a complex operation; compare the proof of the previous lemma.)

This pair of almost complex structures for $Z$ and $\bar{Z}$ corresponds to a 2-plane bundle in $T Z$; compare [9]. According to Thurston [16], any 2-plane bundle can be homotoped to a completely integrable one. Thus $\Gamma$ is $(4,2)$-realizable, and $(n, n-2)$ realizability follows by pulling back this foliation to the total space of iterated $S^{1}$ bundles, as in the proof of the previous lemma.

LEMMA 3.5. If $n \geqslant 5$, then $\Gamma$ is $(n, n-1)$-realizable. If $n \geqslant 7$, then the realization can be done by pulling back the standard 2-dimensional foliation of $S^{3}$.

Proof. If $n \geqslant 7$, then there is an (n-3)-dimensional manifold $X$ with $\pi_{1}(X)=\Gamma$. On $X \times S^{3}$ we pull back the standard foliation from the second factor.

For the general case, let $X$ be a 4-manifold with $\pi_{1}(X) \cong \Gamma$ and $\pi_{2}(X)$ large. We foliate $X \times S^{1}$ by the product foliation $X \times\{t\}, t \in S^{1}$. From this we obtain a 5-manifold $Y$ with $\pi_{1}(Y) \cong \Gamma$ and a codimension 1 foliation $\mathscr{F}$ by surgery on $\{p t\} \times S^{1}$ : foliate $\left(X \backslash D^{4}\right) \times S^{1}$ by Reeb spiraling applied to the above product foliation so that the boundary $S^{3} \times S^{1}$ becomes a leaf, and fill in the foliation of $S^{3} \times D^{2}$ obtained in [1, Corollaire 1]. This shows that $\Gamma$ is $(5,4)$-realizable.

The general case now follows by pulling back $\mathscr{F}$ to the total space of an iterated $S^{1}$-bundle as in the proof of Lemma 3.3.

The Godbillon-Vey invariant referred to in Theorem 2 is the well-known generalization of the definition in [5]. If $\mathscr{F}$ is a $k$-dimensional foliation on an $n$ - 
manifold $X$, then $\mathrm{GV}(\mathscr{F})$ is a cohomology class in $H^{2(n-k)+1}(X, \mathbb{R})$, natural under pullback, which vanishes for elementary foliations like products, bundles and the standard 2-dimensional foliation of $S^{3}$.

LEMMA 3.6. The foliations constructed in Lemmas 3.1-3.5 have trivial Godbillon-Vey invariants.

Proof. The foliations in Lemma 3.2 are products. The foliations in Lemma 3.1 have trivial $\mathrm{GV}(\mathscr{F})$ for dimension reasons, as do the ones in the basic cases $n \in\{4,5\}$ and $k=2$ in Lemmas 3.3 and 3.4. In Lemma 3.5, the basic case for $n \geqslant 7$ is $S^{3}$, so that by the naturality of $\mathrm{GV}(\mathscr{F})$ there is nothing left to prove. The second construction given in the proof of Lemma 3.5 leads to foliations almost without holonomy, so that $\mathrm{GV}(\mathscr{F})=0$ by a result of $\mathrm{G}$. Duminy; see [4].

\title{
References
}

1. N. A'CAMPo, 'Feuilletages de codimension 1 sur les variétés simplement connexes de dimension 5', Comment. Math. Helv. 47 (1972) 514-525.

2. Y. EliaSHBerG, 'Classification of overtwisted contact structures on 3-manifolds', Invent. Math. 98 (1989) 623-637.

3. Y. EliASHBERG, 'Topological characterization of Stein manifolds of dimension $>2$ ', Internat. J. Math. 1 (1990) 29-46.

4. E. GHYS, 'L'invariant de Godbillon-Vey', Séminaire Bourbaki, Astérisque 177-178 (1989) 155-181.

5. C. Godbillon and J. VeY, 'Un invariant des feuilletages de codimension 1', C. R. Acad. Sci. Paris 273 (1971) 92-95.

6. R. E. GOMPF, 'Some new symplectic 4-manifolds', Doğa Mat., to appear.

7. M. Gromov, Partial differential relations, Ergeb. Math. Grenzgeb. (3) (Springer, Berlin, 1986).

8. A. Haffliger, 'Structures feuilletées et cohomologie à valeurs dans un faisceau des groupoìdes', Comment. Math. Helv. 32 (1958) 248-329.

9. F. HIRZEBRUCH and H. HoPF, 'Felder von Flächenelementen in 4-dimensionalen Mannigfaltigkeiten', Math. Ann. 136 (1958) 156-172.

10. F. E. A. Johnson, 'The fundamental group and homological restrictions in dimension four', Preprint.

11. D. KoтsCHICK, 'All fundamental groups are almost complex', Bull. London Math. Soc. 24 (1992) 377-378.

12. D. KotschicK, 'All fundamental groups are almost contact', Bull. London Math. Soc. 24 (1992) 493-494.

13. W. B. R. Lickorish, 'A foliation for 3-manifolds', Ann. of Math. 82 (1965) 414-420.

14. J. Martinet, 'Formes de contact sur les variétés de dimension 3', Proceedings of Liverpool Singularities Symposium II, Lecture Notes in Math. 209 (Springer, New York, 1971).

15. C. MECKERT, 'Forme de contact sur la somme connexe de deux variétés de contact de dimension impaire', Ann. Inst. Fourier 32 (1982) 251-260.

16. W. P. Thurston, 'The theory of foliations of codimension greater than one', Comment. Math. Helv. 49 (1974) 214-231.

17. W. P. Thurston, 'Existence of codimension-one foliations', Ann. of Math. 104 (1976) 249-268.

18. A. Weinstein, 'Contact surgery and symplectic handlebodies', Hokkaido Math. J. 20 (1991) 241-251.

\author{
Mathematisches Institut \\ Universität Basel \\ Rheinsprung 21 \\ 4051 Basel \\ Switzerland
}

